\title{
Urbane Kindheiten zwischen Utopie und Dystopie
}

\author{
Sozial- und erziehungswissenschaftlich informierte Rationalisierungsweisen
}

zum Topos „Kinder in der Stadt“

\author{
Dominik Farrenberg
}

\begin{abstract}
Der vorliegende Beitrag nimmt analytisch in den Blick, wie urbane Kindheiten in der sozialund erziehungswissenschaftlich informierten Rede über Kinder in der Stadt rationalisiert werden. Dies geschieht, indem die spezifischen Rationalisierungsweisen aus der Rede herausgearbeitet werden, welche „Kinder" und „Stadt" diskursiv miteinander relationieren und hierüber topologisch letztlich zwischen Utopie und Dystopie positionieren. Angeleitet von dieser diskurstopologischen Perspektive wird der Topos nach einer kurzen Einleitung zunächst als expliziter Gegenstand empirischer, sozial- und erziehungswissenschaftlicher Forschung in den Blick genommen, bevor dann wesentliche Etappen der Rede über Kinder in der Stadt in Form von chronologischen Schlaglichtern nachgezeichnet werden. Im Anschluss hieran werden die Straßenkindheit und die pädagogische Provinz als vielschichtige und zentrale Narrative dieser Rede analysiert. Schlussgedanken führen die vorliegende Analyse wieder in die Rede ein und reflektieren den Topos „Kinder in der Stadt “ als eine Art "diskursive Heterotopie“.
\end{abstract}

Ersteinreichung: 30. November 2020; Veröffentlichung online: 26. November 2021 An English abstract can be found at the end of the document.

\section{Einleitung}

„Herb und streng ist die Großstadt gegen das Kind vom ersten Lebenstage an. Selbst den Sonnenschein mißt sie ihm kärglich zu. Mit den Blumen und Tieren wird es nicht so vertraut, wie die Jugend in Dorf und Kleinstadt.“ (Tews 1911: 22)

Überdeutlich machte das Eingangszitat bereits zu Beginn des 20. Jahrhunderts auf die Schattenseiten des Aufwachsens von Kindern in der Stadt aufmerksam. Seine Aussage erfährt gerade heute - so ist angesichts der Zunahme des motorisierten Verkehrs, der Bebauungsdichte und der Flächenversiegelung zu vermuten - überwiegend assoziativ-reflexhafte Zustimmung. An dem Zitat überrascht weniger die Aussage, als vielmehr ihr Entstehungskontext. Denn das Werk Großstadtpädagogik (Tews 1911), aus dem es stammt, hebt die Stadt primär als einen anregungsreichen Lern- und Lebensraum für Kinder hervor. So wird ihr zugesprochen, „ein Schulbuch mit Tausenden von Illustrationen" (ebd.: 112) zu sein, welches in pädagogisch bedeutsamer Weise für das Leben bilde. Dieses Plädoyer für eine „Großstadtpädagogik“ wird jedoch - mal unterschwellig, mal explizit 
(hierauf weist das Eingangszitat exemplarisch hin) - von einem steten Rechtfertigungszwang zusammengehalten: Warum sieht sich selbst eine programmatische Fürsprache für ein urbanes Aufwachsen zu einer solchen Verteidigungshaltung veranlasst?

Tatsächlich wird der Diskurs über Kinder in der Stadt spätestens seit dem 18. Jahrhundert überwiegend kritisch geführt. In sozial- und erziehungswissenschaftlich informierten Diskursräumen[1] werden mit dem Topos „Kinder in der Stadt“ zahlreiche, vielfach miteinander verwobene Problematisierungen aufgerufen, wobei sich kulturpessimistische mit bewahrpädagogischen Motiven verschränken (Reutlinger/Brüschweiler 2016: 48 ff.): Kindliches Aufwachsen in der Stadt wird primär als Problem markiert (Karsten/Thunemeyer 1991: 142). Zugleich erhalten diese Diskursräume, die das Wissen über Kinder in der Stadt ordnen, ihre Spannungsmomente und ihr Anregungspotenzial gerade über vereinzelte Positionen, die den vielfältigen Problematisierungen auf eine Weise gegenüberstehen, die das urbane Aufwachsen idealisiert - wie etwa auch in der Tews'schen Großstadtpädagogik. So werden Kinder - ob implizit oder explizit - ebenso als verletzlich, gefährlich, gefährdet, lernend oder sich aktiv Räume aneignend beschrieben, wie die Stadt als gleichermaßen hektisch, verrucht, chancengebend, gefährlich, vielschichtig und facettenreich sowie als Asphaltdschungel, Ballungsraum, Sozialisationsort oder Totalität schraffiert wird.

Der vorliegende Beitrag geht in einer hermeneutisch angelegten Darstellung der Frage nach, wie urbane Kindheiten in der sozial- und erziehungswissenschaftlich informierten Rede über Kinder in der Stadt rationalisiert werden. Analysiert wird damit im Besonderen, welche Bedeutungen und Bedeutungszuweisungen und insofern (Re-)Produktionen von Denkweisen (Kessl 2011: 8) in diesozial-und erziehungswissenschaftliche Redeüber Kinder in der Stadt eingelassen sind. Es wird beschrieben, „wie Zusammenhänge, Unterschiede, Begrenzungen oder Einordnungen gedacht, benannt und gedeutet werden“ (Kessl 2011: 8). Diskurstopologisch perspektiviert sind damit „die Lagebeziehungen der Aussagen in diskursiven Formationen“ (Schreiber 2009: 207) angesprochen, die den Topos „Kinder in der Stadt“ als solchen erst hervorbringen. Rationalisierungsweisen urbaner Kindheiten, die durch diesen Topos aufgerufen werden, verweisen insofern auf die (Re-) Produktion machtvoller Wissensordnungen (Farrenberg 2018: 50 ff.). Erkenntnis- und sozialtheoretisch wird mit dieser Perspektive auf soziale Wirklichkeit als eine insgesamt diskursive Konstruktion abgestellt. Demnach kann auch nicht zwischen Diskurs und „Nicht-Diskurs“ unterschieden werden (etwa in der Annahme eines vom Diskurs unabhängig existierenden „Dahinter“) (Parr 2014: 234; Quadflieg 2008: 94). Abgrenzbewegungen finden vielmehr insofern statt, als der Versuch unternommen wird, die sozial- und erziehungswissenschaftlich informierte Rede über Kinder in der Stadt innerhalb des „diskursiven Gewimmels“ zu identifizieren, sie aus ihm herauszulösen und von anderen Diskursformationen zu separieren. Methodisch angeleitet wird dies von einer systematischen Quellenrecherche. Der bei diesem Vorgehen herausdestillierte Textkorpus bildet die Basis für die in diesem Beitrag vorgelegten Analysen. Er setzt sich zusammen aus den in einschlägigen Bibliothekskatalogen im deutschen Sprachraum verfügbaren sozial- und erziehungswissenschaftlich orientierten Publikationen, die 
entweder Begrifflichkeiten wie „Kind(er) bzw. Kindheit und Stadt“ explizit im Titel führen oder aber eine begrifflich zwar lediglich implizite, inhaltlich jedoch direkte Verbindung zum Topos „Kinder in der Stadt“ aufweisen.[2]

Unter Zugriff dieses diskurstopologischen Fokus wird im Verlauf des Beitrags sichtbar gemacht, wie die Rationalisierungsweisen urbaner Kindheiten letzten Endes zwischen Utopie und Dystopie oszillieren: Einerseits wird der Topos „Kinder in der Stadt“vereinzelt mit utopischen Gehalten aufgeladen. Bis in das machtvolle und polyseme (sprich: vielstimmige) Narrativ der Straßenkindheit lassen sich diese - etwa in Tews' Großstadtpädagogik in Form von idealistisch überhöhten pädagogischen Zielen aufspüren, deren Realisierungsmöglichkeiten jedoch vielfach ungewiss bleiben. Andererseits steht der Topos „Kinder in der Stadt“ mehrheitlich mit dystopischen Gehalten in Verbindung. Neben der bereits erwähnten Polysemie der Straßenkindheit spiegeln diese sich vor allem im Narrativ der pädagogischen Provinz wider, so wie darin ein utopischer Gegenort zur Stadt und ihren (vermeintlichen) Gefahren aufgerufen wird.

Angeleitet von dieser Perspektive wird der Topos „Kinder in der Stadt“ im Folgenden zunächst als expliziter Gegenstand empirischer, sozial- und erziehungswissenschaftlicher Forschung in den Blick genommen. Mit diesem Analyseschritt wird der empirische Forschungsstand im Kontext des Topos exemplarisch umrissen. Dabei wird aufgezeigt, wie das Forschungsfeld maßgeblich von zwei Vergleichshorizonten strukturiert wird, wodurch sich letztlich eine Problematisierung voranschreitender Urbanisierung mit der Problematisierung sozialer Ungleichheiten verschränkt (2.). Unter dem Begriff des „diachronen Streifzugs“[3] werden sodann wesentliche Etappen der sozial- und erziehungswissenschaftlich informierten Rede über Kinder in der Stadt in Schlaglichtern chronologisch nachgezeichnet. In diesem Analyseschritt wird aufgezeigt, wie sich im Verlauf der Rede der Fokus verschiebt von der Stadt als dem das Kind verderbenden Ort schlechthin, zu zunächst konkreten zivilisationsbedingten Veränderungen urbanen Aufwachsens und schließlich zu einzelnen benachteiligten Sozialräumen und einer institutionell begleiteten Kindheit (3.). Im Anschluss hieran werden die Straßenkindheit und die pädagogische Provinz als zentrale und zugleich polyseme Narrative analysiert und in ihren utopischen wie dystopischen Gehalten dargestellt. Über diesen Schritt der Analyse gerät zudem die Frage der Sichtbarkeit von Kindern bzw. Kindheiten im urbanen Raum in den Blick - und damit letztlich auch die Möglichkeit von Überwachung, Disziplinierung und Kontrolle (4.). Schlussgedanken führen die Ergebnisse der vorliegenden Analyse wieder in die Rede ein. Analytisch wird der Topos „Kinder in der Stadt“ hierbei als eine Art „diskursive Heterotopie“ reflektiert (5.).

\section{Kinder in der Stadt als Gegenstand empirischer Forschung}

Blickt man exemplarisch auf empirische Studien sozial- und erziehungswissenschaftlicher Provenienz, die „Kinder in der Stadt“ als Gegenstand von Forschung anvisieren, so ist nach wie vor eine Studie hervorzuheben, die in den Diskursräumen als „Klassiker“ bezeichnet wird: Der Lebensraum des Großstadtkindes (Muchow/Muchow 2012 [1935]). Mit innovativen, quasi ethnographischen Untersuchungsmethoden beschreibt Martha Muchow im 
Spätherbst der Weimarer Republikam Beispiel des Hamburger Stadtteils Barmbek drei analytische Zugänge, in denen Kind und Stadt unter der konzeptionellen Klammer des Lebensraums zueinander relationiert werden.[4] Muchows Konzept des Lebensraums nimmt - wenn auch nicht durchgängig systematisch ausbuchstabiert - die Perspektive eines antiessenzialistischen, relationalen Raumbegriffs vorweg, wie er für zahlreiche aktuelle Zugänge kennzeichnend ist. Über den heute veraltet erscheinenden Begriff rücken Subjekt (Kinder) und materiale Territorialität (Stadt) in eine produktive und dynamische Dialektik ein (vgl. Büttner/Coelen 2012: 199 ff.; Zinnecker 2012 [1978]: 43 ff.).

Indem die Kinder gebeten werden, ihre vertrauten Wege und Orte ebenso auf einem Stadtplan zu schraffieren, wie separat hiervon solche, die ihnen nur durch ein gelegentliches Passieren bekannt sind, adressiert Muchow sie in einem ersten Zugang als ortskundige Expert_innen ihrer Lebensräume. Die kartographierten Lebensräume bilden das Differenzial, anhand dessen die abstrakte Stadt in relevante und irrelevante Areale unterteilt wird - wobei die relevanten Lebensräume nochmals in unmittelbare, den Kindern vertraute Spielräume und mittelbare, fußläufig erkundbare Streifräume differenziert werden. Durch diesen topologisch-territorialen Forschungszugang zerfällt die Stadt in Wohngebiete, Verkehrswege, Grünanlagen, Spielplätze und infrastrukturell bedeutende Einrichtungen wie Schulen und Kaufhäuser. Deren Bedeutung bemisst sich daran, inwiefern sie Aufenthalts- oder Durchgangsorte für die Kinder darstellen. Kinder und (diese parzellierten Orte der) Stadt werden topologisch zueinander in einer Art Lagebeziehung ins Verhältnis gesetzt. Über diese Verzonung und Territorialisierung werden Aufwachsen und Stadtkindheit erst sichtbar als ortsgebundene Phänomene. Untersucht wird der „Raum, in dem das Kind lebt“ (Muchow/Muchow 2012 [1935]: 80).

In einem zweiten Zugang spricht Muchow die Kinder über ein Erfragen ihrer Lieblingsaktivitäten und Spielorte noch einmal stärker als im ersten als Subjekte von Forschung an. Anvisiert wird hier der „Raum, den das Kind erlebt“ (ebd.: 97), also die Qualitäten des urbanen Raumes aus der Erlebensperspektive der Kinder. Inhaltlich favorisieren die Kinder dabei das Spielen auf der Straße.

Mittels verschiedener Beobachtungstechniken geraten die Kinder schließlich in einem dritten Zugang als Akteur_innen in den Blick, die sich die von Erwachsenen konzipierte dinglich-örtliche Umgebung aktiv aneignen und für ihre spezifischen Bedarfe „umleben“ (ebd.: 160). Stadt erscheint in diesem Zugang als „Raum, den das Kind lebt“(ebd.: 106), das heißt als eine den Kindern durch Bebauung und Verkehr gegebene, aber gleichzeitig (um-) gestaltbare, (um-)lebbare material-territoriale Struktur.

Insgesamt betrachtet fungiert Stadt in Muchows Studie als eine Art Kulisse und Bühne. Bildet sie in ihrer Gänze und als abstrakte Idee lediglich die Kulisse, vor welcher der „Lebensraum des Großstadtkindes“ (Muchow/ Muchow 2012 [1935]) prozessiert wird, so treten einzelne Fragmente dieser Kulisse qua ihrer dinglich-örtlichen Gegebenheiten als vorstrukturierte Bühne hervor, auf der die als Akteur_innen positionierten Kinder ihre Aktions-, Wahrnehmungs- und Aneignungsräume aktiv er- und durchleben (Büttner/Coelen 2012: 203 f.).

In Versatzstücken und Variationen instruieren die drei analytischen Zugänge der „Lebensraum-Studie“ bis heute zahlreiche einschlägige 
Studien zu Kindern in der Stadt (exemplarisch von Seggern/Ohrt 1980; Harms/Preissing/Richtermeier 1985; Blinkert 1993; Zeiher/Zeiher 1994; DKHW 2015; Andresen et al. 2016). Mehr oder minder deutlich wird auch die darin eingelassene Verhältnissetzung von Kindern und Stadt somit weiter transportiert und fortgeschrieben. Indes diesen Forschungen zwar gemein ist, dass sie Kinder als von Erwachsenen differente Akteur_innen beschreiben, rückt hingegen die Handlungsmacht der Kinder, die mit diesem Akteur_innen-Status angesprochen ist, in den Hintergrund. Dies gilt insbesondere für jene Studien, die den urbanen Lebensraum der Tendenz nach entweder als unzureichend oder als gefährlich problematisieren (von Seggern/Ohrt 1980; Blinkert 1993; DKHW 2015).

Weiterhin fällt auf, dass die Studien zu Kindern in der Stadt nahezu durchgängig von zwei Vergleichshorizonten strukturiert werden. Zum einen gerät Stadt mehr oder weniger explizit als Kristallisationspunkt zivilisatorischer Wandlungsprozesse in den Blick. Vermittelt über Merkmale wie die Zunahme des Autoverkehrs, die Erhöhung der Bebauungsdichte oder die Entmischung des innerstädtischen Lebens materialisieren sich diese Wandlungsprozesse in veränderten, oftmals als Verschlechterung gelesenen Bedingungen des Aufwachsens. Damit wird der jeweils gegenwärtige Status quo des Forschungsgegenstandes zumindest implizit vor der rekursiven Matrix einer Transformationsbewegung gelesen. Dies erst erlaubt es, das Aufwachsen in der Stadt ortsübergreifend in seiner Qualität zu evaluieren (exemplarisch Blinkert 1993: 9). Zum anderen rückt diskurstopologisch perspektiviert an die Seite dieses diachronen Vergleichs der synchrone Vergleich unterschiedlicher, territorial begrenzter urbaner Räume etwa von Wohnquartieren, Stadtteilen oder ganzen Städten. Der urbane Raum wird hierbei in seiner Konkretion gewissermaßen vor Ort aufgeschlossen und in territorial voneinander unterscheidbare Orte zerlegt, aus denen wiederum differente Qualitäten des Aufwachsens herausgelesen werden. Bei näherer Betrachtung entpuppt sich dieser Vergleichshorizont allerdings als keineswegs rein territorial. Vielmehr ist er Ausdruck von Segregation, in deren Folge soziale und ökonomische Bedingungen des Aufwachsens mit der jeweiligen territorial umgrenzten Ökologie amalgamieren. Exemplarisch zeigt sich dies in folgenden Untersuchungen:

Die historische Studie Stadtgeschichte als Kindheitsgeschichte (Behnken/ du Bois-Reymond/Zinnecker 1989) rekonstruiert unterschiedliche Muster historischer Stadtkindheiten um 1900 am Beispiel der Städte Wiesbaden und Leiden. Diese Muster differenzieren sich entlang von Klassenunterschieden in entsprechend segregierten Wohnquartieren aus.

Die Studien Kinder und Jugendliche in der Großstadt (Harms/Preissing/ Richtermeier 1985) sowie Orte und Zeiten der Kinder. Soziales Leben im Alltag von Großstadtkindern (Zeiher/Zeiher 1994) untersuchen das Aufwachsen von Kindern in jeweils zwei unterschiedlichen Stadtvierteln. Sie machen sichtbar, dass die Freizeit- und Alltagsgestaltung von Kindern je nach Wohnmilieu deutlich divergiert. Sie ist sowohl mit ökologischen als auch mit sozial-ökonomischen Bedingungen verwoben - hier geplante Verabredungen in Zweierfreundschaften im häuslichen Umfeld, dort spontane, ungeplante und teilweise illegale Aktivitäten in losen Cliquen sowie die Teilnahme an Angeboten eines nahen Freizeitheimes. 
Die Studie Raum für Kinderspiel! (DKHW 2015) zeigt auf, dass die Chance von Kindern, in einem Wohngebiet aufzuwachsen, das ,für ihre Lebensqualität und Entwicklungsmöglichkeiten günstig“ (DKHW 2015: 205) ist, in hohem Maße von den sozial-ökonomischen Ressourcen ihrer Herkunftsfamilie abhängt. Diese bilden in Verschränkung mit den ökologischen Bedingungen des jeweiligen Wohnumfeldes eine Art „latentes Curriculum“ - entweder für ein aktives und autonomes oder für ein eher passives und heteronomes Aufwachsen.

Die Studie Urbane Lernräume (Andresen et al. 2016) nimmt Gentrifizierungsprozesse, soziale Diversität sowie den Umgang mit Gefahren im Straßenraum als zentrale Herausforderungen von Eltern in den Blick, sich in einem Innenstadtviertel als Familie zu konstituieren. Dabei werden soziale Ungleichheiten sichtbar, die sowohl die Familien benachteiligen, als auch distinktiv von diesen fortgeschrieben werden.

Empirische Aussagen über Kinder in der Stadt weisen somit vielfach auf die Wirkmacht sozial-ökonomischer Bedingungen hin. Das Aufwachsen in urbanen Räumen ist in erster Linie ein Aufwachsen in unterschiedlichen sozialen Klassen (Ward 1978: 7 ff.). Es differenziert sich auf grundlegende Weise entlang von Klassen- bzw. Schichtzugehörigkeiten aus, die sich zu schichtspezifischen Mustern städtischer Kindheiten typologisieren lassen. Über die beiden aus den empirischen Studien herausgearbeiteten Vergleichshorizonte wird der Topos „Kinder in der Stadt“ gleich zweifach in problematisierender Weise rationalisiert: Die Kritik an einer zunehmenden, das Aufwachsen beeinträchtigenden Urbanisierung verschränkt sich mit einer Kritik an sozialen Ungleichheiten, die als Folge von Segregationsprozessen urbane Kindheiten erkennbar strukturieren (exemplarisch Behnken/du Bois-Reymond/Zinnecker 1989: 12, 22; Ward 1978).

Die Schichtspezifik urbaner Lebensräume, der Vergleich verschiedener urbaner Territorien sowie die rekursive Matrix einer problematisierenden Bezugnahme auf zivilisatorische Wandlungsprozesse stellen Zusammenhänge dar, welche die sozial- und erziehungswissenschaftlich informierte Rede über „Kinder in der Stadt“ - auch über die einzelnen Studien hinaus - insgesamt wirkmächtig ordnen. Im Folgenden werden nun wesentliche Etappen dieser Rede chronologisch in Schlaglichtern nachgezeichnet. In diesem diachronen Streifzug geht es darum, das Geworden-Sein des Topos „,Kinder in der Stadt“ darzustellen. So wie darin Konstanten, Verschiebungen und Brüche der sozial- und erziehungswissenschaftlich informierten Rede sichtbar werden, so wird ebenfalls erkennbar, dass sich der Fokus der Rede in ihrem Fortgang zunehmend zuspitzt und kanalisiert.

\section{Die Rede über Kinder in der Stadt - ein diachroner Streifzug}

Spätestens Mitte des 18. Jahrhunderts dringt die überwiegend kritisch geführte Rede über „Kinder in der Stadt“ in die Öffentlichkeit. Beispielhaft und einflussreich bemängelt Jean-Jacques Rousseau die verdorbene Luft und das zusammengepferchte Gedränge der Stadt. Diesen Mängeln stellt er die reinere Landluft und das freiere, räumlich großzügigere Landleben als positiven Entwurf gegenüber (Rousseau 2010/1762: 62 f.). Weiterhin bringt Rousseau 
das kindliche Aufwachsen in der Stadt zusammen mit dem Vorwurf sexueller Ausschweifungen und krimineller Machenschaften sowie mit einem das Kind verderbenden Umgang schlechthin. Auf geradezu idealtypische Weise ruft sein Erziehungsroman Émile oder über die Erziehung (Rousseau 2010 [1762]) dazu auf, das Aufwachsen von Kindern fern von schädlichen urbanen Einflüssen und Einflüsterungen, im Schutz- und Schonraum der „pädagogischen Provinz“ zu organisieren. Diese Rationalisierungsweise, den Lebensraum Stadt mit einem schlechten, das Kind gefährdenden Einfluss zusammenzudenken, reicht indes weit über Rousseau und seine Perspektive auf die französischen Städte hinaus. Sie entfaltet bis heute Wirkmacht und amalgamiert vielfach mit verklärten Vorstellungen „mittels derer Erwachsene die eigene Kindheit im Rückblick romantisieren"(Reutlinger/Brüschweiler 2016: 48).

In Rekurs auf die Urbanisierungsschübe des 20. Jahrhunderts wird der Topos „Kinder in der Stadt“ dieser Zeit mit weiteren Gefährdungen und Mängeln aufgeladen. Problematisiert wird dabei vor allem, dass synchron zum Wachstum der Großstädte Wohnräume von Arbeits- und Verkaufsräumen geschieden und vom Zentrum an die Peripherie gedrängt werden. Urbanes Aufwachsen verlagert sich somit zu großen Teilen in monofunktional auf Wohnen ausgerichtete Außenbezirke. So hält Johannes Tews im frühen 20. Jahrhundert für Berlin fest, die Großstadt werde „gewissermaßen ausgehöhlt“ (Tews 1911: 4). Auch in der historischen Studie von Imbke Behnken et al. (Behnken/du Bois-Reymond/Zinnecker 1989: 12 f.) findet sich beispielhaft in Wiesbaden und Leiden jene Gleichzeitigkeit städtischer Verdichtung einerseits und einer Zunahme (segregierter) bürgerlicher Vororte und Arbeiter_innenvorstädte andererseits. Dem Band Das Kind in der Stadt (Ward 1978) zufolge markiert die Vorstadt in Europa und Nordamerika gar „das typische Siedlungsmuster des zwanzigsten Jahrhunderts“ (ebd.: 67) schlechthin. Parallel zu dieser Diagnose über den Zerfall eines zuvor als multifunktional interpretierten Stadt(er)lebens gewinnen eine stete Zunahme von Konsum, Lohnerwerbstätigkeit, motorisiertem Verkehr sowie einer dichteren Bebauung an Bedeutung. Diese Veränderungen werden in den sozial- und erziehungswissenschaftlich informierten Diskursräumen spätestens seit den 1960er Jahren als Modernisierungsnebenfolgen problematisiert, die das Aufwachsen von Kindern in der Stadt einschränken und bedrohen (Zeiher 1991: 179). Neben das Bild einer eher diffusen Gefährdung des Kindes im urbanen Raum treten jetzt konkrete modernisierungsbedingte Veränderungen der sozialen Praxis sowie ihrer greifbaren materiell-territorialen Kontexte. Indem sich die Vielfalt des öffentlichen Lebens auf der Straße auflöst, verliert sie auch als Spielraum an Bedeutung. Ein eindrucksvolles Beispiel hierzu gibt der Beitrag „Das Kind und die Straße - von der Stadt zur Anti-Stadt“ (Ariès 1994). Philippe Ariès zeigt darin exemplarisch auf, wie sich Pariser Vororte zwischen 1900 und 1970 dadurch veränderten, dass Bürgersteige verengt, Straßen verbreitert und dem wachsenden Autoverkehr zugewiesen werden (Ariès 1994: 90 ff.). Der Beitrag „Kinder in der Großstadt“ (Zeiher 1995) nimmt Modernisierungsnebenfolgen wie diese auf und beschreibt, wie sich urbane Kindheiten zunehmend in eigens dafür hergerichtete eingezäunte Spielplätze und in private Wohnräume verlagern sowie in weitere kindspezifische institutionelle Umgebungen vor und neben der Schule (ebd.: $39 \mathrm{ff}$.). 
Eine gängige Rationalisierungsweise, die der hier untersuchten Rede inhärent ist, hält fest, dass sich der öffentliche großstädtische Raum im 20. Jahrhundert auch für Kinder sukzessive von einem Aufenthalts- zu einem Durchgangsort transformiert (Ariès 1994: 75; Götzelmann 2017: 123). So ist die Rede davon, dass sich Stadtkindheit von einer Straßenkindheit zu einer „verhäuslichten“ und „verinselten“ Kindheit verschiebt (Behnken/ du Bois-Reymond/Zinnecker 1989: 11 ff.; Behnken/Jonker 1990: 165 f.; Zeiher 1991: 189 ff.; Zinnecker 2001 [1990]). Der Rede nach findet das Aufwachsen nun vermehrt im privaten, begrenzten und vielfach als anregungsarm beschriebenen Binnenraum der Familie statt (Zinnecker 2001 [1990]: 41 f.). An die Stelle eines zusammenhängenden, zonenhaft in Nah- und Streifraum unterscheidbaren Territoriums, in dem das Kind lebt, Stadt erlebt und umlebt (Muchow/Muchow 2012 [1935]) tritt ein loses Netz mit einzelnen Knotenpunkten - etwa der Schule, der Kindertageseinrichtung sowie Wohnungen von Spielpartner_innen und Freizeitangeboten. Der Rede nach erlaubt diese Verinselung kein zusammenhängendes Raumerleben mehr, sondern nur noch ein punktuelles Ortserleben ohne Zugang zu einem territorialräumlichen Dazwischen. Eine Erlebensweise, die durch elterliche Bringund Abholdienste mit dem PKW noch potenziert wird. Über das eigentliche Raumerleben hinaus, so der Befund, fragmentiert die Verinselung auch das Bewusstsein eines einheitlich-konsistenten Selbst sowie die sozialen Beziehungen (Zeiher 1991: 189).

Parallel zu den Diagnosen urbaner Modernisierungsprozesse und den analytischen Rekonstruktionen veränderter, hierauf reagierender Kindheitsmuster nehmen insbesondere in den 1970er bis 1990er Jahren weitere empirische und programmatische Beiträge, aber auch Praxisberichte zu. In diesen, teilweise auf der Ebene von Kommunalpolitik und Stadtplanung verorteten Beiträgen wird das konkrete urbane Territorium zunächst nun in kinderfreundliche und kinderfeindliche Aspekte und Faktoren zergliedert. Hierauf aufbauend werden schließlich Empfehlungen für eine kinderfreundliche Stadt formuliert (exemplarisch Blinkert 1993; Harms/ Preissing/Richtermeier 1985; Kißler/Keller-Ebert 1994; Difu 1991; TU Braunschweig 1980). Dem Befund einer autogerechten Stadt wird die Utopie einer kindergerechten Stadt gegenübergestellt, die durch geeignete politische Steuerungsmaßnahmen zur Realutopie werden soll. Unter anderem wird diskutiert, neue Bauvorhaben analog zu Umweltverträglichkeitsprüfungen einer „Kinderverträglichkeitsprüfung“ zu unterziehen (Blinkert 1993: 225; Difu 1991). Vielfach wird das programmatischeZiel einer kinderfreundlichen Stadt mit der Idee von Beteiligungs- und Mitbestimmungsmöglichkeiten für die lokalen Kinderöffentlichkeiten verknüpft - etwa über die Implementierung von Kinderbeauftragten, -büros, -versammlungen und -parlamenten (Difu 1991; Schröder 1995). Gleichzeitig wird postuliert, eine kinderfreundliche Stadt stelle quasi unweigerlich zugleich eine insgesamt bürger_innenfreundliche Stadt dar (exemplarisch Pickmann 1980: 192; Feldtkeller 2001: 88). Implizit scheint in dieser Diskursfigur jener „romantische Kindheitsmythos“ (Baader 2004) durch, der seit Beginn des 19. Jahrhunderts das Kind (quasi-)religiös und ästhetisch auflädt, es als rein und unverdorben glorifiziert und es in seiner als natürlich gegeben verstandenen Unbekümmertheit und Phantasie zum idealisierten Vorbild für eine 
deformierte Erwachsenengesellschaft macht (ebd.: 418). In dieser Vorstellung vom Kind spiegelt sich die (real-)utopische Potenzialität, die Stadt bürger_innenorientiert in einen vitaleren, grüneren und das Wohlergehen aller befördernden Wohn- und Lebensraum umzugestalten. Vielerorts werden kommunale Modellprojekte initiiert, die ihre Überzeugungskraft implizit aus einer Rationalisierungsweise ziehen, wonach eine am Prädikat der Kinderfreundlichkeit ausgerichtete kommunale Stadtentwicklung und -planung den urbanen Raum zu einer lebenswerten Stadt für alle transformieren kann.

Jedoch bleiben die Modellprojekte dieser Zeit mehr Projekte als Modelle. So wie die Rede über Kinder in der Stadt - gemessen am Publikationsaufkommen - insbesondere in den 1970er bis 1990er Jahren stark anschwillt, so ist sie in darauf folgenden Jahrzehnten bis heute nahezu verstummt, zumindest auf den ersten Blick. Der Reformeifer scheint absorbiert worden zu sein von einer an anderen Rationalitäten ausgerichteten Realität. Jedenfalls wird die bis hierhin skizzierte Rede heute lediglich punktuell fortgesetzt, etwa in Gestalt des Sammelbandes Kind sein in der Stadt (Fischer/Rahn 2017) oder der vom Deutschen Kinderhilfswerk herausgegebenen Studie Raum für Kinderspiel! (DKHW 2015). Kann es sein, dass die bis hierhin skizzierte Rede analog dazu, wie die Stadtkindheit von der Sichtbarkeit auf der Straße in eine zunehmend unsichtbare Verhäuslichung sowie in ein institutionelles Aufwachsen eingewandert ist (Blinkert 1993: 23; Müller 1980: 49; Muri Koller 2017: 49), dorthin mitgewandert ist?

Bei genauerer Betrachtung zeigt sich durchaus eine Verschiebung bzw. Kanalisierung der Diskurslinien. Etwa seit den 1990er Jahren wird urbanes Aufwachsen vor allem im Kontext von Segregation und benachteiligten Stadtquartieren sowie den institutionellen Bedingungen des Aufwachsens akzentuiert. Publikationstitel wie Aufwachsen und Lernen in der sozialen Stadt. Kinder und Jugendliche in schwierigen Lebensräumen (Bruhns/ Mack 2001) oder Quartier macht Schule (Fritsche/Rahn/Reutlinger 2011) verweisen ebenso darauf wie politische Programme und Konzepte, etwa das Bund-Länder-Programm „Stadtteile mit besonderem Entwicklungsbedarf - die soziale Stadt“ (Difu 1999) oder das Positionspapier des Bundesjugendkuratoriums „Neue Bildungsorte für Kinder und Jugendliche“(Bundesjugendkuratorium 2004). Mit dieser diskursiven Verschiebung wird die Rede über „Kinder in der Stadt“ nun ein weiteres Mal konkretisiert und zugespitzt. Angesprochen werden nun nicht mehr die urbane soziale Praxis und deren materiell-territorialen Kontexte schlechthin. Stattdessen werden einerseits die schwierigen Bedingungen des Aufwachsens in benachteiligten, von Segregation gekennzeichneten und mitunter als „soziale Brennpunkte“ markierten Wohnquartieren fokussiert. Andererseits geraten Schulen und weitere institutionelle Orte städtischen Aufwachsens stärker in den Blick. Diese beiden Foki laufen zusammen in einer wiederentdeckten, politisch wie fachwissenschaftlich-professionell gestaltbaren Steuerungsgröße: dem sozialen Nahraum.

Programmatisch sowie fachwissenschaftlich-konzeptionell wird diese Möglichkeit kommunaler Steuerung über die Leitbegriffe „Sozialraum“ und „Bildungslandschaften“ ausbuchstabiert (Dirks/Kessl 2012: 512 ff.; Brüschweiler/Falkenreck 2019). Beiden Konzepten ist die Machbarkeitsvorstellung inhärent, unter der Prämisse von Vernetzung und Kooperation Ressourcen lokaler Bildungs-, Gesundheits- und Sozialeinrichtungen sowie 
der Bürger_innen eines Stadtteils aktivieren und Potenziale arrangieren zu können (Koch/Schulz 2018: 44). Hierdurch soll „eine optimale Begleitung von Kindern und Jugendlichen, durch gesundes Aufwachsen und gutes Lernen vor Ort“ (Brüschweiler/Falkenreck 2019: 423) möglich werden (Dirks/ Kessl 2012: 512; Kessl/Reutlinger 2011). Kritisiert wird indes, dass die Fokussierung auf solche Synergieeffekte die unterschiedlichen Ziele, Arbeitsweisen, Kindheits- und Bildungsverständnisse der beteiligten Akteur_innen außer Acht lässt (z. B. von Schule und Kinder- und Jugendhilfe). Zudem wird auf unzulässige Weise eine Planbarkeit von Bildungsprozessen suggeriert (Koch/ Schulz 2018). Weiterhin sieht sich die Konzentration auf den Sozialraum der Kritik ausgesetzt, dass sämtliche Probleme territorialisiert und auf ein sodann als problematisch markiertes Quartier bezogen werden, auch wenn Probleme primär sozial oder ökonomisch bedingt sind bzw. mit dem Wohnund Wirtschaftsstandort einer ganzen Stadt oder einer gesamten Region zusammenhängen (Häussermann 2011: 277; Dirks/Kessl 2012: 515 f.; Kessl/ Reutlinger 2011).

Jedenfalls wird der Topos „Kinder in der Stadt“ in der Rede von Sozialräumen und Bildungslandschaften nun mehrheitlich indirekt angesprochen. Indem diese zuvorderst auf bildungsbezogene Aktivitäten, die Infrastruktur des sozialen Nahraums sowie eine Vernetzung der unterschiedlichen Akteur_innen und Institutionen abstellt, wird das Kind diskursiv dezentriert und die Stadt in verschiedene lokale Sozial- und Bildungsräume zergliedert. Die Stadt als Ganzes tritt als Abstraktum in den Hintergrund. Unbehelligt von den benachteiligenden Lebenslagen in einzelnen Quartieren und der dortigen, das Aufwachsen institutionell rahmenden Infrastruktur, wird die Stadt in ihrer Gesamtheit weder als Verursacherin von Problemlagen noch als Problemlöserin adressiert. Im Unterschied dazu gerät das parzellierte Territorium benachteiligter Quartiere auf diffuse Weise in den Blick - sowohl als Kumulationspunkt von Konflikten und Problemen als auch, vermittelt über den Taschenspielertrick einer nahräumlichen Vernetzung von Bildungsangeboten und der Aktivierung von Ressourcen, als deren Lösung (Kessl/Reutlinger 2011: 286 f.; Dirks/Kessl 2012: 515). Ähnlich diffus erscheint auch die polyseme Position, die infolge der Konzentration auf den urbanen Nahraum dem Kind zugeschrieben wird. So werden in der Rede vom benachteiligten Quartier gleichermaßen die Muster eines gefährdeten wie eines gefährlichen Kindes aufgerufen (Jenks 2005 [1996]: 62 ff.). Das Konzept der Bildungslandschaften ist hingegen auf das Muster des aktiven, wissbegierigen Entdeckerkindes angewiesen (Elschenbroich 2001), das seine Bildungsprozesse im Zusammenspiel vernetzter Bildungsorte in seinem Nahraum selbst organisiert (Koch/Schulz 2018). Letzteres Muster fungiert unreflektiert oftmals als anthropologische Vorannahme jener „neuen Bildungsdebatte“, die seit der Jahrtausendwende nicht nur die gesellschaftliche Aufmerksamkeit auf Bildung zentriert, sondern zugleich auch das Bildungsgeschehen kommunalisiert, territorialisiert sowie mit Stadt(teil) entwicklung verschränkt. Insofern stellt es einen diskursiven Begründungszusammenhang für die Programmatik kommunaler Bildungslandschaften dar (Million/Heinrich/Coelen 2015; Brüschweiler/Falkenreck 2019).

Zusammengefasst verschiebt sich im Verlauf der sozial- und erziehungswissenschaftlich informierten Rede der Fokus von der Stadt als einem das Kind 
insgesamt verderbenden Ort zunächst zu konkreten zivilisationsbedingten Veränderungen im urbanen Raum und einer darauf abgestimmten sozialen Praxis, bevor schließlich mehrheitlich nur noch punktuell einzelne benachteiligte Sozialräume und ein institutionell begleitetes Aufwachsen anvisiert werden. Den vielen in die Rede eingelassenen Problematisierungen von Stadt stehen dabei vorwiegend Idealisierungen gegenüber, die das Kind in seiner Verletzlichkeit hervorheben.

In diesem diachronen Streifzug durch die sozial- und erziehungswissenschaftlich informierte Rede über Kinder in der Stadt kristallisieren sich zwei zentrale, polyseme und ambivalente Narrative heraus, die den Topos auf wirkmächtige Weise strukturieren - gewissermaßen querliegend zu den vorliegend skizzierten Etappen. Gemein ist ihnen, dass sie beide implizit wenn auch unterschiedlich konnotiert - auf die Sichtbarkeit von Kindern bzw. Kindheiten abstellen: Angesprochen sind damit die Narrative der Straßenkindheit und der pädagogischen Provinz. Diese beiden Narrative halten die Rede gewissermaßen zusammen. Sie werden im Folgenden einer tiefergehenden Analyse unterzogen.

\section{Straßenkindheit und pädagogischer Provinz - zentrale Narrative des Topos „Kinder in der Stadt“}

\section{Das Narrativ der Straßenkindheit}

Grundsätzlich ist das Wissen, das sich aus dem Topos „Kinder in der Stadt“ speist, eng verknüpft mit der Frage der Sichtbarkeit von Kindern im urbanen Raum. Die sozial- und erziehungswissenschaftlich informierte Rede greift unmittelbar auf das Sichtbar-Werden oder eben auch Nicht-SichtbarWerden urbaner Kindheiten zurück. Im Rückgriff auf die rekursive Matrix eines zivilisatorischen Wandels wird in diesem Zusammenhang vor allem das Verschwinden der einstigen, überwiegend sichtbaren Straßenkindheit problematisiert. In diesem Narrativ der Straßenkindheit finden sich zwei negative Gegenhorizonte eingelassen: die vielfache Verlagerung von Stadtkindheit in städtische Vororte und mehr noch die zunehmende Etablierung einer überwiegend unsichtbaren „verhäuslichten Kindheit“, die sich mehrheitlich im Wohnungsinneren abspielt (Müller 1980: 49). Hierüber wird die ohnehin ambivalente und polyseme Position, die die Straßenkindheit im Diskursfeld ausfüllt, aktualisiert und weiter fortgeschrieben (Zinnecker 2001 [1979]: 48).

Zum einen wird mit dem Begriff der Straßenkindheit idealisierend die Mitgliedschaft in einem nachbarschaftlichen Kinderkollektiv aufgenommen, das draußen auf der Straße, an der frischen Luft und in Bewegung einen anregungsreichen Spiel- und Sozialisationsort vorfindet. Diese Idealisierung war mancherorts, insbesondere in den Arbeiter_innenvierteln, bis in die 1960er Jahre hinein zugleich Realität, wie historische Rekonstruktionen von Stadtkindheiten im 20. Jahrhundert in Wiesbaden und Leiden exemplarisch zeigen (Behnken/du Bois-Reymond/Zinnecker 1989: 12 f.). Mit der Straßenkindheit verbindet sich der Gedanke eines Aufwachsens in einem sozialen Biotop. Dieses fördert über das soziale Miteinander in der Gruppe Selbstbehauptung, Unterordnung und Gemeinschaftssinn. Die aktive Teilhabe von Kindern an der Straßenöffentlichkeit ermöglicht 
Vergesellschaftung. Gemäß diesem Ideal einer „Pädagogik der Straße“ (Tews 1911: 114) wird die Straße als Lernfeld, als pädagogischer Ort besonderer Qualität verstanden (Zinnecker 2001 [1979]: 51; Fuhs 2001: 138; Tews 1911: 108 ff.; Forsell 2014: 204 f.). Mit dieser Rationalisierungsweise verbindet sich geradezu utopisch die Hoffnung, dass die Straßenkinder „die Samen eines neuen kulturellen Lebens in sich tragen könnten: einer anderen Sozialisierung und einer freieren Gesellschaft“ (Forsell 2014: 205). Im semantischen Gefolge dieser Idealisierung erscheint Straßenkindheit bis heute als positiver Antipode zu einer verhäuslichten Kindheit, welche die Rede über Kinder in der Stadt vielfach mit Bewegungsmangel, Anregungsarmut, dem Fehlen sozialer Kontakte, der intensiven Nutzung digitaler Medien sowie einer verstärkten Konsumorientierung assoziiert (exemplarisch Muri Koller 2017: 50; Zinnecker 2001 [1990], DKHW 2015).

Zum anderen verengt sich im Verlauf der sozial- und erziehungswissenschaftlich informierten Rede - in etwa parallel zum Befund einer zunehmenden Verhäuslichung städtischer Kindheiten - der Begriff der Straßenkindheit. Während das bürgerliche Kind sich in einem gewissen Sinne seitjeher - das heißt in etwa seit dem 18. Jahrhundert - weitestgehend in Privaträumen verorten lässt, folgen ihm in einer langen „Privatisierungsbewegung“ (Ariès 1994: 75) dorthin zunächst Kinder aus kleinbürgerlichen Milieus sowie später aus Arbeiter_innenmilieus (Behnken/du Bois-Reymond/Zinnecker 1989: 11 ff.; Behnken/Jonker 1990: 165 ff.; Forsell 2014: 215; Zinnecker 2001 [1990]: 37 ff.). Die Straße entvölkert sich schrittweise. Sie wird mehr und mehr zum Durchgangsort und ,als Aufenthaltsort unmoralisch“ (Ariès 1994: 75). „Der Übergang von einer straßen- zu einer haus- und familienorientierten Kindheit vollzieht sich“ (Behnken/Jonker 1990: 166) in einer zunehmenden Segregation der sozialen Klassen „von oben nach unten“" (Behnken/Jonker 1990: 166, Hervorhebung im Original). Schließlich umreißt der Begriff der Straßenkindheit nur noch einzelne und als abweichend markierte Straßenkinder. „Ausreißer und Trebegänger“ (Jogschies 1998: 193), die ,jugendpolitisch und jugendhilfepolitisch einen Handlungsbedarf" (ebd.: 195) signalisieren. So wird das Narrativ der Straßenkindheit schleichend zum Synonym für ein Aufwachsen in ärmlichen, teilweise erbärmlichen Verhältnissen (Ariès 1994: $79 \mathrm{ff}$.) sowie zur Metapher für ein „gegenpädagogisches Milieu“ (Zinnecker 2001 [1979]: 48). Diese lineare, gesellschaftstheoretisch gerahmte Rationalisierungsweise des Narrativs ist aus historischer Perspektive betrachtet allerdings weit weniger eindeutig. Denn die Spur von „Straßenkindern“, die als Tagedieb_innen und Tagelöhner_innen auf dem „rauen Pflaster“ der Straße ihr Überleben zwischen Kriminalität und Armut sichern müssen (Cunningham 2006: 204 ff.; Forsell 2014: 203), lässt sich mindestens bis ins 16. Jahrhundert zurückverfolgen (Cunningham 2006: 164 ff.). Analog hierzu lassen sich ebenso für die polizeilichen, caritativen und sozialpädagogischen Eingriffe, die im 18. Jahrhundert verstärkt einsetzten, um die Kinder „von der Straße zu holen“, sie in öffentlichen Erziehungseinrichtungen zu versorgen und dort für eine Zukunft ,jenseits der Straße“ auszurüsten (Ariès 1994: 81), historische Vorläuferpraktiken und Institutionen beschreiben. Hiervon zeugen Berichte etwa aus Paris, Stockholm sowie aus weiteren Städten in Preußen (Cunningham 2006: 165 f.; Bühler-Niederberger 2003: 176). 
In einer Zusammenschau der Ambivalenz und Polysemie des hier dargestellten Narrativs zeigt sich anstelle einer eindeutigen und linearen Verschiebung der semantischen Gehalte des Begriffs der Straßenkindheit vielmehr ein ambivalentes Oszillieren zwischen problematisierenden und idealisierenden Rationalisierungsweisen. Verbunden hiermit werden zugleich divergierende Muster von Kindheit aufgerufen: Problematisierende Rationalisierungsweisen sehen die Straße als einen Umschlagplatz, an dem sich das zuvorderst verwahrloste und von Armut betroffene gefährdete Kind sukzessive transformiert in ein gaunerisches, verdorbenes gefährliches Kind (Ariès 1994: 85 f.; Bühler-Niederberger 2010: 36; Cunningham 2006: 164 f., 204 ff.). Idealisierende Rationalisierungsweisen erteilen hingegen dem verletzlichen und verdorbenen Kind eine Absage und stellen ihm das lernende, mit Handlungsmacht ausgestattete Kind entgegen (Forsell 2014: 204). Entsprechend ist die Straßenkindheit bis heute gleichermaßen Zielpunkt von Utopien wie Dystopien. Zudem wird sie zu einer Art Vexierbild, in dem sich die mit ihr aufgerufenen Problematisierungen zwar stets auf die Stadt richten, jedoch mal die Öffentlichkeit der Straße, mal die Privatheit des Wohnungsinneren adressieren.

Diese Dichotomie von Öffentlichkeit und Privatheit macht auf wesentliche Bedeutungshorizonte des Narrativs aufmerksam: Noch in der mittelalterlichen Stadt spielte nicht nur das Kind auf der Straße. Vielmehr war jene Drehund Angelpunkt des sozialen Lebens schlechthin. Philippe Ariès verdeutlicht dies beispielhaft an Kunstwerken aus unterschiedlichen Epochen, die den Archetypus der italienischen Stadt porträtieren (Ariès 1994: 75 ff.). Ab dem Beginn der Moderne driften die Aktionsräume von Erwachsenen und Kindern - Arbeit und Spiel -jedoch in einem jahrhundertelang andauernden Prozess mehr und mehr auseinander. Erst hierdurch kristallisiert sich Kindheit als eigene Lebensphase heraus (Ariès 2014). Kinder werden zu einem Erziehungsprojekt, dessen ,zentrale Mechanismen der Scholarisierung und Familialisierung [...] als aufteilende Verräumlichung von Kindheit zwischen gesellschaftlich-staatlicher wie privater Sphäre“(Bollig 2018: 112) alternative Sozialisationsorte jenseits der Straße markieren. Neben die Öffentlichkeit der Straße treten im Zivilisationsprozess der Moderne zunächst der private Raum familialer Intimität sowie später der halb-öffentliche, staatlich kontrollierte Raum der Schule (Zinnecker 2001: 10; Mierendorff 2013; Ariès 2014): Diese „eingezäunte[n] Kinderghetto[s]“ verschärfen „die Trennung zwischen der Welt der Erwachsenen und der der Kinder" (Ward 1978: 87). Zivilisationstheoretisch gesprochen ist Straßenkindheit somit die „Kennzeichnung einer Differenz“ (Zinnecker 2001: 10): Diese „bezeichnet das vormoderne wilde, unzivilisierte Kind - im Kontrast zum zivilisierten Familien- und Schulkind“ (ebd.). Schlussendlich stellte sich eine „Trennung von Privatheit und Öffentlichkeit im heutigen Sinne" (Müller 1980: 49) erst ein, nachdem die moderne Familie im 18. Jahrhundert eine „Mauer des Privatlebens zwischen sich und die Gesellschaft schob“ (Ariès 2014: 562). Was bedeutet diese „Mauer der Privatheit" nun im Hinblick auf die Sichtbarkeit städtischer Kindheiten?

Auf diese Frage bezogen liegt eine Pointe darin, dass das Einziehen der zivilisierenden Trennung zwischen Öffentlichkeit und Privatheit (Elias 2017 [1939]) zwar vordergründig zu einer Unsichtbarkeit von Kindern in der urbanen Öffentlichkeit geführt hat. Gleichzeitig haben jedoch 
halb-öffentliche institutionelle Erziehungsräume wie Schule und Kindertageseinrichtung oder der private Erziehungsraum der Familie eine spezifisch veränderte Sichtbarkeit erhöht, in der auf besondere Weise die Möglichkeit von Überwachung, Disziplinierung und Kontrolle angelegt ist (Bühler-Niederberger 2003: 175 ff.; Fegter 2014: 521). Diese neuen Sichtbarkeitsräume einer verhäuslichten Familienkindheit sowie zeitlich versetzt einer institutionell gerahmten Bildungskindheit etablieren sich in den westeuropäischen Städten des 19. und 20. Jahrhunderts sukzessiv und großflächig. Sie werden zum Gegenentwurf einer öffentlich sichtbaren Straßenkindheit. Im Folgenden werden sie in der Utopie der „pädagogischen Provinz“ reflektiert, jenem zweiten zentralen Narrativ, das den Topos „Kinder in der Stadt“" wirkmächtig strukturiert. Darin enthalten ist eine zweite Pointe: Die pädagogische Provinz - ursprünglich konzipiert als Gegenentwurf zu einem Aufwachsen in der Stadt - verlagert sich selbst zunehmend in die Stadt. Sie wird Teil von Stadt und kann somit nicht mehr ohne Weiteres als deren utopisches Gegenüber in Stellung gebracht werden.

\section{Das Narrativ der pädagogischen Provinz}

Als diskursiver Ausgangspunkt des Narrativs der pädagogischen Provinz gilt gemeinhin die bereits erwähnte Problematisierung eines städtischen Aufwachsens bei Rousseau. Indem dieser die schädlichen und lasterhaften Einflüsse durchgängig auf den urbanen Raum projiziert, macht er die Stadt zum topologischen Ort für eine grundsätzliche Staats- und Gesellschaftskritik, vor deren Hintergrund er seine negative Erziehung ausformuliert (Rousseau 2010 [1762]: 28, 280, 642 f., 766 ff.). Die Stadt wird so zum Sinnbild und zur verräumlichten Manifestation allen die gute Natur des Kindes bedrohenden gesellschaftlichen Übels. In der Stadt lassen sich der Fortschritt der Moderne, der Sitz der bürgerlichen Klasse, die Etablierung gesellschaftlicher Institutionen, ja das gesellschaftliche Leben schlechthin verorten. Dieses Zusammenfallen von Gesellschaft und Urbanität im Verständnis einer Gefahr für die Erziehung führt mehr oder weniger zwangsläufig zu Rousseaus Devise „raus aus der Stadt“. Der Gegenentwurf einer Erziehung auf dem Land ist nicht nur Stadtflucht, sondern vor allem Schutz vor den das Kind verderbenden gesellschaftlichen Einflüssen. In der „pädagogischen Provinz"vermag sich Rousseaus Erziehungsideal leichter durchzusetzen als in der flirrenden Stadt, die mit ihren vielfältigen Gelegenheiten und Verlockungen Aufmerksamkeit zerstreut und Ablenkung stiftet. Kehrseite der Idee eines provinzial angelegten Schutzraumes ist die Möglichkeit einer besseren Kontrolle bzw. Kontrollierbarkeit von Aufwachsen und Erziehung des jungen Menschen (ebd.: 136f.). Vordergründig setzt Rousseaus Devise gegen den urbanen Raum die bis heute wirksame Rationalisierungsweise in Gang, Aufwachsen und Erziehung im ländlichen Raum zu organisieren. Damit ist jedoch hintergründig eine weitere, mindestens genauso wirkmächtige Rationalisierungsweise verbunden: die Abschirmung des kindlichen Aufwachsens vor dem öffentlichen, gesellschaftlichen Raum. In Rousseaus Devise „raus aus der Stadt" verbindet sich die Utopie eines naturnahen Aufwachsens im ländlichen Raum mit der Utopie einer erzieherischen Kontrolle über das Aufwachsen an einem eingrenzbaren und überblickbaren Ort. 
Aufgehoben findet sich dieser stadt- und gesellschaftskritische, bewahrpädagogische Argumentationszusammenhang in der auf Johann Wolfgang von Goethe zurückgehenden Metapher der „pädagogischen Provinz“ (Goethe 1962 [1821/1829]).[5] In Goethes Erzählung ist die pädagogische Provinz ein utopischer Ort, der Aufwachsen und Erziehung befreit von sämtlichen beeinträchtigenden Einflüssen und Faktoren ermöglicht. Versuche, dieses Ideal in die Realität umzusetzen, finden sich mit expliziter Bezugnahme hierauf vor allem in Gestalt der reformpädagogischen Landerziehungsheime (Oelkers 2017). Hier kommen die beschriebenen utopischen Gehalte, die bereits in Rousseaus Devise angelegt sind, erneut zum Ausdruck. In den stadtfernen Landerziehungsheimen geht es zum einen darum, Kinder „vor den Gefahren der Großstadt“ (ebd.: 128) zu schützen - ausgehend von der Vorstellung, „dass es auf dem Lande gesünder zugehe als in der Stadt und deswegen die neue Erziehung dort stattfinden müsse“ (ebd.). Zum anderen zeichnet sich die „abgeschiedene pädagogische Provinz“ (ebd.: 125), die in Anlehnung an Rousseau „von wohlmeinenden Erziehern kontrolliert werden“ (ebd.) müsse, auch in der reellen Ausgestaltung der Landerziehungsheime durch Kontroll- und Überwachungsmechanismen aus. Diese zielen letztlich auf die Schaffung eines hermetisch abgeriegelten und Abhängigkeitsverhältnisse stiftenden Raums ab. Was als Schutz- und Schonraum konzipiert war, geriet nicht selten zu einem Gefängnis.

Weitreichender noch lässt sich inzwischen empirisch beobachten, dass einzelne Aspekte des Narrativs mehr oder minder flächendeckend in die Betreuungs-, Bildungs- und Erziehungssysteme gegenwärtiger bürgerlicher Gesellschaften eingewandert sind. Insbesondere die deutliche Abgrenzung von der Außenwelt, mit der pädagogische Einrichtungen vielerorts das Aufwachsen vor dem Blick der Öffentlichkeit abschirmen, es hierüber regulieren, kontrollieren sowie ihre spezifischen Eigenlogiken entfalten, lassen jene als reale pädagogische Provinzen erscheinen. Bis heute lässt sich dieser Aspekt der regulierenden und Kontrolle ermöglichenden Abschirmung in Schulen und Kindertageseinrichtungen nahezu durchgängig aufzeigen (exemplarisch Farrenberg 2018: $141 \mathrm{ff}$.) - und zwar unabhängig von territorialen Markierungen wie Stadt und Land. Damit verlagern sich nun Spuren der pädagogischen Provinz in die Stadt hinein.

Die Realutopie einer pädagogischen Provinz, die Rousseau zunächst als verräumlichten Antipoden gegen die bürgerliche Gesellschaft konzipierte (Rousseau 2010 [1762]: $18 \mathrm{ff}$.), geriet paradoxerweise - etwa in Gestalt der Landerziehungsheime - zunehmend zu einer dezidiert bildungsbürgerlichorientierten, oftmals gar elitären Angelegenheit (Oelkers 2017: 117 ff.). Bereits in den Pflegefamilien-Programmen „für verwahrloste oder delinquente Kinder" (Cunningham 2006: 210), die im 19. Jahrhundert eingerichtet wurden, um jene „aus dem ungesunden Milieu der Städte“ (ebd.: 210) zu entfernen, ,in ländliche Gegenden“ (ebd.) zu verpflanzen und somit „,vor den Städten und vor ihren unzulänglichen Familien zu retten" (ebd.: 211) zeichnete sich ab, was bis heute zunehmend konkreter werden sollte: Nicht mehr das Abstraktum Gesellschaft, sondern vielmehr bestimmte, nicht-bürgerliche Milieus und Familien sind es, vor denen das Kind in der pädagogischen Provinz geschützt werden soll. Es ist der Fokus auf ein schadenfrei-behütetes Moratorium der Kindheit, der das Narrativ der 
pädagogischen Provinz originär mit bildungsbürgerlichen Motiven zusammenbringt. Denn eine derartige Aufmerksamkeitszentrierung auf das Kind war in den unteren Klassen lange Zeit weder ideell rationalisierbar, noch in den dort herrschenden Bewältigungsbedingungen des Alltags realisierbar (ebd.: 95 f., 271 f.; Donzelot 1980: 35 f.).

Diese behütende Konnotation des Narrativs wird besonders in jenen Strategien deutlich, mit denen im 19. Jahrhundert in Frankreich versucht wurde, die unteren Schichten nach bürgerlichem Vorbild zu familialisieren, um ihren in Teilen als zügel- und sittenlos erachteten Lebenswandel ebenso zu unterbinden, wie die Verwahrlosung ihrer Kinder (Donzelot 1980). Sozialund ordnungspolitische Maßnahmen zum Aufbau von Privateigentum, zur Förderung sozialen Aufstiegs, zur Absicherung des Wohlergehens und zur Unterstützung bei Betreuungs-, Bildungs- und Erziehungsaufgaben sollten dabei Anreize setzen und zu einer Selbstführungsweise anleiten, die sich ,am gesellschaftlichen Ideal eines moralisch-sittlichen, ökonomischnachhaltigen und kulturell-bildungsbürgerlichen Familienlebens ausrichtet“ (Farrenberg 2020: 56). Teil dieser gouvernementalen Ordnungsstrategie einer Erziehung zu Privatheit und Familiensinn war seinerzeit auch eine bauliche Neuordnung der Armenviertel in französischen Großstädten. Die damalig neu eingerichteten Sozialwohnungen wurden exakt so konzipiert, dass der Wohnraum gerade „groß genug ist, um hygienisch zu sein, klein genug, damit nur die Familie ihn bewohnen kann, und so unterteilt, daß die Eltern die Kinder überwachen können. Man verlangt von der Wohnung ein Gegenstück zur Schule bei der Überwachung der Kinder zu werden“ (Donzelot 1980: 57 f.). Über ebendiese Kontrolle, die der private, nach außen abgeschirmte Erziehungsraum der Familie ermöglicht, wird das Narrativ der pädagogischen Provinz erneut mit aufgerufen. Auch hierüber wandert die pädagogische Provinz in die Stadt mit ein.

Wie bis hierhin deutlich wurde, ist das Narrativ weder selbst neutral gegenüber der Kategorie der Klasse, noch sind es die Rationalisierungsweisen, die metaphorisch daran anknüpfen. Dennoch kennzeichnet der darin aufgerufene Schutz- und Schonraum als Ordnungsschema Kindheit insgesamt (Mierendorff 2013: 59).

Zum einen spannt sich dieser Raum über das potenziell gefährdete Kind des Bürgertums. „Die bürgerliche Familie legt um das Kind einen sanitären Sicherheitsgürtel, der sein Ausdehnungsfeld begrenzt“ (Donzelot 1980: 60). Unschuld und Verletzlichkeit des Kindes (aber auch seine Bedeutung für den Fortbestand der Familie) verlangen danach, es ins Zentrum einer wohlbehütenden Umgebung zu stellen, die Lernen und Entwicklung befördert; ins Zentrum binnenfamilialer Aufmerksamkeit schlechthin (Jenks 2005 [1996]: 64 ff.; Schmid 2014: 42 ff.). In einem pädagogischen Modell der „geschützten Befreiung“ (Donzelot 1980: 60, Hervorhebung im Original) wird das Kind sowohl in seiner Entwicklung gefördert als auch „durch eine diskrete Überwachung kontrolliert“ (ebd.). Hieran anschließend lässt sich die Stadtflucht junger Familien in die Vororte und Außenbezirke als Versuch deuten, eine solche wohlbehütende wie kontrollierbare Umgebung für das kindliche Aufwachsen realisieren zu können - in den überschaubaren Strukturen der in parzellierte Privatflächen aufgeteilten Aneinanderreihung von Eigenheimen (Ariès 1994: 89; Ward 1978: 34). Wenige Praktiken lassen 
den auf das Wohl des Kindes zentrierten Lebensentwurf der modernen Kleinfamilie so deutlich werden, wie der vielfach um den Zeitpunkt der Familiengründung praktizierte Auszug aus der Stadt - ein Wohnortwechsel unter dem idealisierten Vorzeichen der pädagogischen Provinz.

Zum anderen umfasst der Schutz- und Schonraum das potenziell gefährliche Kind aus unteren Schichten, das infolge seiner Devianz gegenüber den allgemeinen, bürgerlich konnotierten Erziehungsvorstellungen begrenzt, kontrolliert und diszipliniert werden muss (Jenks 2005 [1996]: 62 ff.). In einem pädagogischen Modell der „überwachten Freiheit“(Donzelot 1980: 60, Hervorhebung im Original) wird versucht, ein „Übermaß an Freiheit und Straßenleben“ (ebd.) zu begrenzen und „das Kind in Räume stärkerer Überwachung, Schule oder Elternhaus, zurückzuführen“ (ebd.). Zu einer faktischen Behebung sozialer Ungleichheiten oder gar zu einer Nivellierung von Klassenunterschieden dürften solche Strategien - also die Umsiedlung benachteiligter Kinder von der Straße in die pädagogische Provinz einer verhäuslichten Familienkindheit oder einer institutionell gerahmten Bildungskindheit - hingegen nur in überschaubarem Maße beitragen. Nicht nur stellt sich mit der Verlagerung des Aufwachsens ,in den privaten Raum der Familie [...] auch eine Privatisierung der Reproduktion sozialer Ungleichheit“ (Bühler-Niederberger 2003: 181) ein. Auch vermögen öffentliche Betreuungs-, Bildungs- und Erziehungseinrichtungen oftmals gerade dann nicht zur einer Reduktion sozialer Ungleichheiten beizutragen, wenn sich ihre Regeln, Anforderungen und Normen als disparat zu jenen der Herkunftsfamilie erweisen (Betz 2007).

Insgesamt ist festzuhalten, dass die bürgerliche Distinktion gerade über das Zusammenspiel klassenspezifisch differenter Kindheitsmuster perpetuiert wird. Jenseits dieser Klassenspezifik und zugleich tieferliegender eint beide mit dem Narrativ der pädagogischen Provinz korrespondierenden Kindheitsmuster die Rationalisierungsweise eines zumindest impliziten Bedarfes an Überwachung und Kontrolle (Jenks 2005 [1996]: 60 ff.). In letzter Konsequenz wird das Narrativ so seines utopischen Gehaltes beraubt: Der gleich doppelt paradoxe Umstand, weder Gegenentwurf zur Stadt noch zur bürgerlichen Gesellschaft sein zu können, reduziert das Narrativ auf sich zuspitzende Weise auf Überwachung und Kontrolle, wodurch seine dystopischen Gehalt schließlich überdeutlich werden.

\section{Schlussgedanken - der Topos „Kinder in der Stadt“ als „diskursive Heterotopie“?}

In einem großen Bogen lässt sich hinter der sozial- und erziehungswissenschaftlich informierten Rede über den Topos „Kinder in der Stadt“ eine sich intensivierende disziplinierende Sozialkontrolle erkennen, die in der öffentlich sichtbaren Straßenkindheit ihren Ausgang nimmt und in der veränderten Sichtbarkeit halb-öffentlicher und privater pädagogischer Provinzen bis heute immer weiter perfektioniert wird (Bühler-Niederberger 2003; Fegter 2014: 521).

Aufder Vorderbühne hat sich die überwiegend problematisierende Rede über Kinder in der Stadt - wie holzschnittartig gezeigt werden konnte - in drei Etappen gewandelt: Zunächst wurden eher abstrakte gesellschaftliche 
Gefahren thematisiert, um eine Stadtflucht in die pädagogische Provinz zu begründen. Im Verlauf der Rede konkretisierte sich diese abstrakte Gefährdung. Die Zunahme an motorisiertem Verkehr, Bebauungsdichte und Gentrifizierung wurden spätestens ab den 1980er Jahren als Merkmale einer sich dystopisch verändernden sozialen Praxis im urbanen Raum gelesen, der die Utopie einer kinderfreundlicheren Stadt entgegengestellt wurde. Schließlich fokussierte sich die Rede auf einzelne benachteiligte Stadtteile sowie auf ein institutionell betreutes Aufwachsen entlang der Steuerungsprogrammatik von Sozialräumen und Bildungslandschaften.

Aufder Hinterbühne hingegen, dies machen die hier vorgelegten Analysen deutlich, haben sich die utopischen Szenarien des Aufwachsens - sei es der als anregungsreich und ganzheitlich betrachtete städtische Lebensraum der Straße oder die vor gesellschaftlichen Zwängen und den Gefährdungen von Stadt und Straße schützende Provinz - in ihr dystopisches Gegenteil verwandelt, in einen disziplinierenden Überwachungsraum. Dessen gegenwärtige Aggregate werden mitunter auch gemäß einer ökonomistischen Verwertungslogik - genauer gesagt sozialinvestiv und humankapitalistisch - kalibriert: Nicht nur die Rationalisierungsweisen des urbanen Aufwachsens haben sich gewandelt, auch der gesellschaftliche Stellenwert des Kindes selbst hat sich verschoben. So wird das Kind insbesondere über bildungs- und sozialpolitische Steuerungsmechanismen zunehmend rationalisierbar als Humankapitalträger in einem Sozialinvestitionsstaat (Olk 2007). Überwachung und Kontrolle verknüpfen sich dabei mit dem Motiv der Notwendigkeit gesellschaftlicher, (national-)ökonomischer Zukunftssicherung.

Sichtbar wird der hier herausgearbeitete Zusammenhang zwischen dem Topos „Kinder in der Stadt“ und einer Intensivierung der das Kind disziplinierenden Sozialkontrolle erst über die Analyse der beiden polysemen und ambivalenten Narrative der Straßenkindheit und der pädagogischen Provinz. Diese strukturieren die Rede auf zentrale Weise. Auch wenn urbanes Aufwachsen sich heute kaum noch als Straßenkindheit realisiert und Stadtkindheit gegenwärtig vor allem als Familienkindheit im privaten Raum sowie als Bildungskindheit im halb-öffentlichen Raum prozessiert wird, entfalten die mit dem Narrativ der Straßenkindheit verbundenen Rationalisierungsweisen nach wie vor ihre Wirkmacht. Wie eine Art Joker lässt sich die „Karte Straßenkindheit“ in der Rede spielen, sei es, um vor Verwahrlosung und weiteren Gefahren der Straße zu warnen oder um die Freiheit des Erlebnis- und Begegnungsraums Straße als idealisierenden Gegenentwurf zum als begegnungs- und erlebnisärmer rationalisierten Rückzug ins Private aufzurufen. Zudem haben sich Spuren der pädagogischen Provinz in den urbanen Raum geschlichen, in Gestalt der verhäuslichten Familienkindheit und der institutionell gerahmten Bildungskindheit. In die Städte hineinverlagert schützen, fördern und kontrollieren die machtvollen Rationalisierungsweisen dieses Narrativs das Aufwachsen in entsprechend eingezäunten Kinderspielplätzen, Schulen, Kindertageseinrichtungen sowie in den Privatwohnungen der Stadtquartiere (Zeiher 1995: 42 f.). Heutige Stadtkindheit zeigt sich damit mehrheitlich als betreute Kindheit (Bollig 2018; Zeiher 1995). Die Schule, aber auch sozialpädagogische Einrichtungen der einstigen Jugendfürsorge und Jugendpflege und heutigen Kinder- und 
Jugendhilfe (etwa Kindertageseinrichtungen, Jugendzentren, Tagesgruppen und Erziehungsheimen) bilden seit dem 19. Jahrhundert ein sich bis heute ständig erweiterndes und ausdifferenzierendes Netz institutioneller Bildungs-, Betreuungs- und Erziehungsorte, welche die Erziehung durch die Familie und die Straße zu ergänzen, in Teilen zu unterstützen und in Teilen zu ersetzen versuchen (Thole 2012: $23 \mathrm{ff}$.).

Weiterhin ist in die Rede eine mehr oder minder fortlaufende Auseinandersetzung mit sozialen Ungleichheiten eingelassen. Das konkrete Aufwachsen im urbanen Raum wird nicht nur dahingehend problematisiert, dass es maßgeblich von einer klassen- bzw. schichtspezifischen Segregation sowie von ungleichen sozioökonomischen und sozialökologischen Bedingungen charakterisiert ist. Auch spiegelt die Rede - insbesondere in Gestalt ihrer beiden zentralen Narrative - selbst einen (bildungs-) bürgerlichen Bias wider: In ihr kommt eine implizite, von einer bürgerlich konnotierten Blickrichtung angeleitete Normalfolie von Stadtkindheit zu Vorschein (Schmidt 2016), die angesichts der heterogenen und benachteiligenden Bedingungen urbanen Aufwachsens vielfach gebrochen wird.

Die vorliegende Analyse legt kondensiert heruntergebrochen frei, wie die sozial- und erziehungswissenschaftlich informierte Rede über den Topos „Kinder in der Stadt“, angeleitet durch ihre zentralen Narrative und die empirischen Forschungen, auf die sie Bezug nimmt, letztlich auf ein klassenbzw. schichtspezifisch segregiertes, urbanes Aufwachsen in intensivierten Überwachungsräumen abstellt.

Diskurstopologisch perspektiviert ließ sich herausarbeiten, wie mit dem Topos teilweise idealisierende, überwiegend jedoch problematisierende Rationalisierungsweisen aufgerufen werden, die in der Rede einander kreuzen, sich wechselseitig verstärken oder auch unverbunden nebeneinanderstehen. Deutlich wurde zudem, wie der Topos im Geflecht dieser Rationalisierungsweisen spannungsreich und wirkmächtig aufgeladen wird sowohl mit utopischen als auch mit dystopischen Gehalten. Erst das polyseme Zusammenwirken dieser Rationalisierungsweisen entbirgt die Widersprüche und Brüche, die in der Rede enthalten sind. Schematisch sichtbar werden diese in einer Art gegensätzlicher Gleichzeitigkeit: So markiert die Straßenkindheit einen anregungsreichen Lernort jenseits häuslicher und institutioneller Kontrolle (Zinnecker 2001 [1979]: 53), während sie gleichzeitig ein Sinnbild für Gefährdung, Verrohung und Verwahrlosung ist. Auch wird die Stadt in der Rede einerseits als abstrakte Totalität adressiert, während andererseits vielfach Segregation, soziale Ungleichheit und Gestaltungsmangel angesprochen werden (Götzelmann 2017: 123). Ebenso verschränken sich der problematisierende Blick auf die Stadt und der idealisierende Blick auf das Kind, um einerseits soziale Probleme zu territorialisieren (Kessl/Otto 2007), während andererseits diskursiv nur schwer artikulierbare Probleme, Veränderungsbedarfe und Wünsche der Erwachsenen auf das Kind projiziert werden. Schließlich ist die Schutz versprechende Utopie der pädagogischen Provinz unlängst in die Stadt eingerückt. Hier kontrolliert sie das urbane Aufwachsen in Gestalt dystopischer Überwachungsräume.

Zusammengenommen hat es den Anschein, als würden sich Bedeutungen und Bedeutungszuweisungen des Topos vor allem aus diesem Schema einer gegensätzlichen Gleichzeitigkeit generieren. Michel Foucault hat für 
dieses Schema einmal den schillernden Begriff der Heterotopie eingeführt. Heterotopien beschreiben auf metaphorische Weise (Gegen-)Orte, deren Topologie von zahlreichen Brüchen und Widersprüchen gekennzeichnet ist. Sie verweisen auf reale Orte, liegen aber gleichzeitig außerhalb dieser. Heterotopien bringen unterschiedliche, einander eigentlich ausschließende Orte zusammen. Ferner stehen sie „in Verbindung mit zeitlichen Brüchen“ (Foucault 2006 [1967]: 324) und entlarven Realitäten als Illusion (ebd.: 320ff.). In diskurstopologischer Analogie hierzu lässt sich der Topos „Kinder in der Stadt", so wie er parallel utopische und dystopische Rationalisierungsweisen ermöglicht und seine Bedeutungen und Bedeutungszuweisungen vom Schema einer gegensätzlichen Gleichzeitigkeit zusammengehalten werden, als eine Art „diskursive Heterotopie“ reflektieren.

\section{Endnoten}

[1] Mit dieser sprachlich etwas sperrigen Bezugnahme auf „sozial- und erziehungswissenschaftlich informierte Diskursräume“ wird vorliegend versucht, den verschiedenen, ineinanderlaufenden disziplinären Herkünften Rechnung zu tragen, welche für die vorliegend analysierte Rede über Kinder in der Stadt kennzeichnend sind. Noch einmal anders abstrahiert - an dieser Stelle danke ich Marc Schulz für seine wertvollen Anmerkungen - lässt sich die Architektur des Beitrags gleichzeitig auch als eine Denkbewegung deuten, die erziehungswissenschaftliche Antworten auf vornehmlich sozialwissenschaftliche Analysen formuliert.

[2] Die Recherche des Textkorpus wurde technisch durch das Onlineportal der Digitalen Bibliothek (DigiBib) unterstützt. Aus Gründen des Umfangs können vorliegend nicht sämtliche Quellen des Korpus angeführt werden. Vielfach stehen die im Beitrag genannten Quellen exemplarisch für weitere, welche die hier analysierten Rationalisierungsweisen und Narrative auf ähnliche Weise stützen. Dieses Vorgehen ist methodisch insofern zulässig, als die diskurstopologische Analyse nach den verschiedenen Bedeutungen und Bedeutungszuweisungen fragt, die mit dem Topos „Kinder in der Stadt“ verbunden sind, während hingegen den einzelnen Autor_innenschaften und Publikationsorten jenseits ihrer prinzipiellen Aufnahme in den systematisch recherchierten Textkorpus keine Relevanz beigemessen wird. Demnach ist auch eine nationalstaatliche Zuordnung von Quellen bzw. Autor_innenschaften vorliegend irrelevant. Die Relevanzsetzung erfolgt allein durch die Zugehörigkeit der Quellen zum Textkorpus, so dass somit auch in die deutsche Sprache übersetzte, internationale Publikationen von z. B. Philippe Ariès, Hugh Cunningham und Colin Ward Berücksichtigung fanden. Gleichzeitig werden stadt- oder kindheitstheoretische Schriften nicht systematisch mitberücksichtigt, sondern lediglich dann, wenn sie die Auseinandersetzung mit dem Topos „Kinder in der Stadt“ konturieren.

[3] Vorliegend wird die Bezeichnung „diachron“ von ihrer originären Bedeutung der Zeitlichkeit von Sprache herausgelöst und stärker auf die Konstanten, Verschiebungen und Brüche der diskursiven Rede in der Zeit bezogen (Landwehr 2014).

[4] Erste Vorarbeiten zu der Studie führte Martha Muchow bereits 1928 und 1929 durch. Die methodisch angeleitete empirische Hauptanalyse erfolgte zwischen 1930 und 1932. Im September 1933 wählte Martha Muchow angesichts der nationalsozialistischen Repressalien, denen sie sich als Mitarbeiterin am Psychologischen Institut des jüdischen Psychologen William Stern ausgesetzt sah, den Freitod. Nach ihrem Tod führte ihr jüngerer Bruder Hans Heinrich Muchow die hinterlassenen Protokolle, Manuskripte und weiteren Aufzeichnungen für eine Publikation der Studie zusammen. Die Veröffentlichung erfolgte 1935 (vgl. Muchow/Muchow 2012 [1935]: 76 f.; Zinnecker 2012 [1978]: 21 f.).

[5] Auch wenn die Idee der pädagogischen Provinz in Rousseaus Erziehungsphilosophie bereits vorweggenommen wird, ist die Wortschöpfung selbst auf Johann Wolfgang von Goethe zurückzuführen. 


\section{Autor_innen}

Dominik Farrenberg ist Sozialpädagoge. Er arbeitet zu Subjekt- und Ordnungsbildungen in sozialstaatlich gerahmten Erziehungs- und Fürsorgeverhältnissen.

d.farrenberg@katho-nrw.de

\section{Literatur}

Andresen, Sabine / Fegter, Susann / Iranee, Nora / Bütow, Elena (2016): Doing urban family. Städtischer Raum und elterliche Perspektive auf Kindheit. In: Zeitschrift für Pädagogik 62/1, 34-47.

Ariès, Philippe (1994): Das Kind und die Straße. Von der Stadt zur Anti-Stadt (Kinder und Umgebung). In: Freibeuter 60, 75-94.

Ariès, Philippe (2014): Geschichte der Kindheit. In: Philippe Ariès (Hg.), Geschichte der Kindheit. München: Deutscher Taschenbuch Verlag, 45-588.

Baader, Meike Sophia (2004): Der romantische Kindheitsmythos und seine Kontinuitäten in der Pädagogik und in der Kindheitsforschung. In: Zeitschrift für Erziehungswissenschaft 7/3, 416-430.

Behnken, Imbke / du Bois-Reymond, Manuela / Zinnecker, Jürgen (1989): Stadtgeschichte als Kindheitsgeschichte. Lebensräume von Großstadtkindern in Deutschland und Holland um 1900. Opladen: Leske + Budrich.

Behnken, Imbke / Jonker, Agnes (1990): Straßenspielkindheit in Wiesbaden und Leiden. Historische Ethnographie und interkultureller Vergleich. In: Imbke Behnken (Hg.), Stadtgesellschaft und Kindheit im Prozeß der Zivilisation. Konfigurationen städtischer Lebensweise zu Beginn des 20. Jahrhunderts. Opladen: Leske + Budrich, 163-200.

Betz, Tanja (2007): Formale Bildung als „Weiter-Bildung“ oder „Dekulturation“ familialer Bildung? In: Christian Alt (Hg.), Kinderleben - Start in die Grundschule. Band 3: Ergebnisse aus der zweiten Welle. Wiesbaden: VS Verlag für Sozialwissenschaften, 163-187.

Blinkert, Baldo (1993): Aktionsräume von Kindern in der Stadt. Eine Untersuchung im Auftrag der Stadt Freiburg. Pfaffenweiler: Centaurus.

Bollig, Sabine (2018): Making Places. Zu den Orten und Räumen „betreuter Kindheiten“. In: Tanja Betz / Sabine Bollig / Magdalena Joos / Sascha Neumann (Hg.), Institutionalisierungen von Kindheit. Childhood studies zwischen Soziologie und Erziehungswissenschaft. Weinheim: Beltz Juventa, 111-128.

Bruhns, Kirsten / Mack, Wolfgang (Hg.) (2001): Aufwachsen und Lernen in der sozialen Stadt. Kinder und Jugendliche in schwierigen Lebensräumen. Opladen: Leske + Budrich.

Brüschweiler, Bettina / Falkenreck, Mandy (2019): Bildungsorte und Bildungslandschaften als sozialraumbezogenes Handlungsfeld. In: Fabian Kessl / Christian Reutlinger (Hg.), Handbuch Sozialraum. Grundlagen für den Bildungs- und Sozialbereich. Wiesbaden: Springer VS, 419-433.

Bühler-Niederberger, Doris (2003): Natürliche Räume oder Architektur der Kindheit: die „Verortung“ der Kinder zwischen generationalem Ordnen und Individualisierung. In: Neue Praxis 33/2, 171-187.

Bühler-Niederberger, Doris (2010): Organisierte Sorge für Kinder, Eigenarten und Fallstricke - eine generationale Perspektive. In: Doris Bühler-Niederberger / Johanna Mierendorff / Andreas Lange (Hg.), Kindheit zwischen fürsorglichem Zugriff und gesellschaftlicher Teilhabe. Wiesbaden: VS Verlag für Sozialwissenschaften, 17-41.

Bundesjugendkuratorium (2004): Neue Bildungsorte für Kinder und Jugendliche. https:// www.bundesjugendkuratorium.de/stellungnahmen (letzter Zugriff am 20.11.2020).

Büttner, Kester / Coelen, Thomas (2012): Kritische Würdigung der „Lebensraum“-Studie aus dem Blickwinkel der aktuellen Methodendiskussion. Ungereimtheiten, Nachwirkungen und Perspektiven. In: Imbke Behnken / Michael-Sebastian Honig (Hg.), Der Lebensraum des Großstadtkindes. Weinheim: Beltz Juventa, 198-211.

Cunningham, Hugh (2006): Die Geschichte des Kindes in der Neuzeit. Düsseldorf: Artemis $\&$ Winkler.

Difu - Deutsches Institut für Urbanistik (1991): Kinder in der Stadt. Berlin.

Difu - Deutsches Institut für Urbanistik (1999): Vorbereitungspapiere zum Bund-LänderProgramm „Stadtteile mit besonderem Entwicklungsbedarf - die soziale Stadt“. 
DKHW - Deutsches Kinderhilfswerk (2015): Raum für Kinderspiel! Eine Studie im Auftrag des Deutschen Kinderhilfswerkes über Aktionsräume von Kindern in Ludwigsburg, Offenburg, Pforzheim, Schwäbisch-Hall und Sindelfingen. Berlin: LIT.

Dirks, Sebastian / Kessl, Fabian (2012): Räumlichkeit in Erziehungs- und Bildungsverhältnissen. In: Ullrich Bauer / Uwe H. Bittlingmayer / Albert Scherr (Hg.), Handbuch Bildungs- und Erziehungssoziologie. Wiesbaden: Springer VS, 507-525.

Donzelot, Jacques (1980): Die Ordnung der Familie. Frankfurt am Main: Suhrkamp.

Elias, Norbert (2017 [1939]): Über den Prozeß der Zivilisation. Beide Bände. Frankfurt am Main: Suhrkamp.

Elschenbroich, Donata (2001): Weltwissen der Siebenjährigen. Wie Kinder die Welt entdecken können. München: Kunstmann.

Farrenberg, Dominik (2018): RegierungsSpielRäume. Eine Ethnographie über Praktiken der Herstellung des Kindergartenkindes. https://voado.uni-vechta.de/handle/21.11106/120 (letzter Zugriff am 28.2.2018).

Farrenberg, Dominik (2020): Bildungsorientierungen oder Ordnungsbildungen? Forscherische Blicke auf Bildung in Familien in der meritokratischen Wissensgesellschaft. In: Michael Hermes / Miriam Lotze (Hg.), Bildungsorientierungen. Theoretische Reflexionen und empirische Erkundungen. Wiesbaden: Springer, 47-65.

Fegter, Susann (2014): Räumliche Ordnungen guter Kindheit. Zum Potenzial praxeologischer Zugänge für die Child-Well-being-Forschung. In: Zeitschrift für Pädagogik 6o/4, 520-534.

Feldtkeller, Andreas (2001): Stadtentwicklung und Soziale Arbeit - Aufgaben der kommunalen Planung. In: Kirsten Bruhns / Wolfgang Mack (Hg.), Aufwachsen und Lernen in der sozialen Stadt. Kinder und Jugendliche in schwierigen Lebensräumen. Opladen: Leske + Budrich, 73-88.

Fischer, Sabine / Rahn, Peter (Hg.) (2017): Kind sein in der Stadt. Bildung und ein gutes Leben. Opladen u. a.: Barbara Budrich.

Forsell, Håkan (2014): Die großstädtische Kindheit. In: Meike Sophia Baader / Florian Eßer / Wolfgang Schröer (Hg.), Kindheiten in der Moderne. Eine Geschichte der Sorge. Frankfurt/New York: Campus, 190-225.

Foucault, Michel (2006 [1967]): Von anderen Räumen. In: Jörg Dünne / Stephan Günzel / Hermann Doetsch / Roger Lüdeke (Hg.), Raumtheorie. Grundlagentexte aus Philosophie und Kulturwissenschaften. Frankfurt am Main: Suhrkamp, 317-329.

Fritsche, Caroline / Rahn, Peter / Reutlinger, Christian (2011): Quartier macht Schule. Die Perspektive der Kinder. Wiesbaden: VS Verlag für Sozialwissenschaften.

Fuhs, Burkhard (2001): Räume der Kinder - Platz für Kinder. In: Kirsten Bruhns / Wolfgang Mack (Hg.), Aufwachsen und Lernen in der sozialen Stadt. Kinder und Jugendliche in schwierigen Lebensräumen. Opladen: Leske + Budrich, 131-146.

Goethe, Johann Wolfgang von (1962 [1821/1829]): Die Pädagogische Provinz. Aus „Wilhelm Meisters Wanderjahre oder die Entsagenden“. In: Wilhelm Flitner (Hg.), Goethes Pädagogische Ideen. Die Pädagogische Provinz nebst verwandten Texten. Düsseldorf und München: Helmut Küpper, 11-39.

Götzelmann, Arnd (2017): Über Kinderleben in der Stadt: Widersprüche und Impulse. In: Sabine Fischer / Peter Rahn (Hg.), Kind sein in der Stadt. Bildung und ein gutes Leben. Opladen u. a.: Barbara Budrich, 119-138.

Harms, Gerd / Preissing, Christa / Richtermeier, Adolf (1985): Kinder und Jugendliche in der Großstadt. Berlin: Fortbildungsinstitut für die pädagogische Praxis.

Häussermann, Hartmut (2011): Das Bund-Länder-Programm „Stadtteile mit besonderem Entwicklungsbedarf - die Soziale Stadt“. In: Heinz-Jürgen Dahme / Norbert Wohlfahrt (Hg.), Handbuch kommunaleSozialpolitik. Wiesbaden:VSVerlag für Sozialwissenschaften, 269-279.

Jenks, Chris (2005 [1996]): Childhood. Abingdon: Routledge.

Jogschies, Peter (1998): Die Lebenswelt von Straßenkindern in Deutschland. Überlegungen und Erkenntnisse im Verlauf eines Forschungsprojektes. In: Christel Adick (Hg.), Straßenkinder und Kinderarbeit. Sozialisationstheoretische, historische und kulturvergleichende Studien. Frankfurt am Main: IKO-Verlag für Interkulturelle Kommunikation, 193-209.

Karsten, Maria-Eleonora / Thunemeyer, Bernd (1991): Kinder zwischen Stadt und Land. In: Ulf Preuss-Lausitz (Hg.), Kriegskinder, Konsumkinder, Krisenkinder. Zur Sozialisationsgeschichte seit dem Zweiten Weltkrieg. Weinheim/Basel: Beltz, 142-149. 
Kessl, Fabian (2011): Die Analyse von Rationalisierungspraktiken als Perspektive sozialpädagogischer Forschung. https://www.uni-due.de/imperia/md/content/biwi/kessl/ rationalisierungspraktiken_kessl_2011_.pdf (letzter Zugriff am 27.7.2015).

Kessl, Fabian / Otto, Hans-Uwe (Hg.) (2007): Territorialisierung des Sozialen. Regieren über soziale Nahräume. Opladen: Barbara Budrich.

Kessl, Fabian / Reutlinger, Christian (2011): Sozialraumorientierung in der Kinder- und Jugendhilfe. In: Heinz-Jürgen Dahme / Norbert Wohlfahrt (Hg.), Handbuch kommunale Sozialpolitik. Wiesbaden: VS Verlag für Sozialwissenschaften, 280-293.

Kißler, Mechtilde / Keller-Ebert, Cornelia (1994): Kinder und Jugendliche in der Stadt. Zur Lebenssituation von Familien unter besonderer Berücksichtigung der Stadt Köln. Köln: Deutscher Gemeindeverlag.

Koch, Sandra / Schulz, Marc (2018): Bildungslandschaft. In: Franz Kasper Krönig (Hg.), Kritisches Glossar Kindheitspädagogik. Weinheim/Basel: Beltz Juventa, 40-47.

Landwehr, Achim (2014): Diachronie. In: Daniel Wrana / Alexander Ziem / Martin Reisigl / Martin Nonhoff / Johannes Angermüller (Hg.), DiskursNetz. Wörterbuch der interdisziplinären Diskursforschung. Berlin: Suhrkamp, 69-70.

Mierendorff, Johanna (2013): Frühe Kindheit und Wohlfahrtsstaat. Wandel des Musters früher Kindheit. In: Sektion Sozialpädagogik und Pädagogik der frühen Kindheit (Hg.), Konsens und Kontroversen. Sozialpädagogik und Pädagogik der frühen Kindheit im Dialog. Weinheim/Basel: Beltz Juventa, 58-72.

Million, Angela / Heinrich, Anna Juliane / Coelen, Thomas (2015): Schnittfelder zwischen Stadtentwicklung und Bildungswesen. In: Thomas Coelen / Anna Juliane Heinrich / Angela Million (Hg.), Stadtbaustein Bildung. Wiesbaden: Springer VS, 1-19.

Muchow, Martha / Muchow, Hans Heinrich (2012 [1935]): Der Lebensraum des Großstadtkindes. In: Imbke Behnken / Michael-Sebastian Honig (Hg.), Der Lebensraum des Großstadtkindes. Weinheim/Basel: Beltz Juventa, 75-16o.

Müller, Peter (1980): Kinderspiel auf Wohnstraßen - Defizite und Möglichkeiten. In: TU Braunschweig (Hg.), Seminar für Planungswesen. Braunschweig: TU Braunschweig, 47-72.

Muri Koller, Gabriela (2017): Gesellschaftsräume: Wo sind die Kinder? Argumente für eine kindergerechte Struktur- und Prozessqualität im Alltag. In: Sabine Fischer / Peter Rahn (Hg.), Kind sein in der Stadt. Bildung und ein gutes Leben. Opladen u. a.: Barbara Budrich, 49-63.

Oelkers, Jürgen (2017): Goethe, Fichte und die Landerziehungsheime. In: Claude Haas / Johannes Steizinger / Daniel Weidner (Hg.), Goethe um 1900. Berlin: Kulturverlag Kadmos, 117-138.

Olk, Thomas (2007): Kinder im „Sozialinvestitionsstaat“. In: Zeitschrift für Soziologie der Erziehung und Sozialisation 27/1, 43-57.

Parr, Rolf (2014): Diskurs. In: Clemens Kammler / Rolf Parr / Ulrich Johannes Schneider (Hg.), Foucault-Handbuch. Leben - Werk - Wirkung. Stuttgart/Weimar: J.B. Metzler, 233-237.

Pickmann, Heinz (1980): Aktion „Kinderfreundliche Stadt Herten“. In: TU Braunschweig (Hg.), Seminar für Planungswesen. Braunschweig: TU Braunschweig, 173-194.

Quadflieg, Dirk (2008): Sprache und Diskurs. Von der Struktur zur différance. In: Stephan Moebius / Andreas Reckwitz (Hg.), Poststrukturalistische Sozialwissenschaften. Frankfurt am Main: Suhrkamp, 93-107.

Reutlinger, Christian / Brüschweiler, Bettina (2016): Sozialgeographien der Kinder - eine Spurensuche in mehrdeutigem, offenem Gelände. In: Rita Braches-Chyrek / Charlotte Röhner (Hg.), Kindheit und Raum. Opladen u. a.: Barbara Budrich, 35-64.

Rousseau, Jean-Jacques (2010 [1762]): Émile oder über die Erziehung. Köln: Anaconda.

Schmid, Pia (2014): Die bürgerliche Kindheit. In: Meike Sophia Baader / Florian Eßer / Wolfgang Schröer (Hg.), Kindheiten in der Moderne. Eine Geschichte der Sorge. Frankfurt/New York: Campus, 42-71.

Schmidt, Friederike (2016): Pädagogische Wahrnehmbarkeitsräume. Historisch-anthropologische Annäherungen an die Verfasstheit pädagogischer Blicke. In: Friederike Schmidt / Marc Schulz / Gunther Graßhoff (Hg.), Pädagogische Blicke. Weinheim/Basel: Beltz Juventa, 54-71.

Schreiber, Verena (2009): Raumangebote bei Foucault. In: Georg Glasze (Hg.), Handbuch Diskurs und Raum. Theorien und Methoden für die Humangeographie sowie die sozialund kulturwissenschaftliche Raumforschung. Bielefeld: transcript, 199-212. 
Schröder, Richard (1995): Kinder reden mit! Beteiligung an Politik, Stadtplanung und Stadtgestaltung. Weinheim: Beltz.

TU Braunschweig (Hg.) (1980): Seminar für Planungswesen. Braunschweig: TU Braunschweig.

Tews, Johannes (1911): Großstadtpädagogik. Leipzig: B. G. Teubner.

Thole, Werner (2012): Die Soziale Arbeit - Praxis, Theorie, Forschung und Ausbildung. In: Werner Thole (Hg.), Grundriss Soziale Arbeit. Ein einführendes Handbuch. Wiesbaden: VS Verlag für Sozialwissenschaften, 19-70.

von Seggern, Hille / Ohrt, Tim (1980): Möglichkeiten einer kindgerechten Gestaltung des engeren Wohnumfeldes in dicht bebauten innerstädtischen Wohngebieten. In: TU Braunschweig (Hg.), Seminar für Planungswesen. Braunschweig: TU Braunschweig, 121-146.

Ward, Colin (1978): Das Kind in der Stadt. Frankfurt am Main: Goverts.

Zeiher, Hartmut J. / Zeiher, Helga (1994): Orte und Zeiten der Kinder. Soziales Leben im Alltag von Großstadtkindern. Weinheim/München: Juventa.

Zeiher, Helga (1991): Die vielen Räume der Kinder. Zum Wandel räumlicher Lebensbedingungen seit 1945. In: Ulf Preuss-Lausitz (Hg.), Kriegskinder, Konsumkinder, Krisenkinder. Zur Sozialisationsgeschichte seit dem Zweiten Weltkrieg. Weinheim/Basel: Beltz, 176-195.

Zeiher, Helga (1995): Kinder in der Großstadt: Raumorganisation und soziale Integration. In: Widersprüche 58, 39-48.

Zinnecker, Jürgen (2001): Einleitung. In: Jürgen Zinnecker, Stadtkids. Kinderleben zwischen Straße und Schule. Weinheim/München: Juventa, 9-24.

Zinnecker, Jürgen (2001 [1979]): Straßensozialisation. Ein Kapitel aus der Geschichte von Kindheit und Pädagogik. In: Jürgen Zinnecker, Stadtkids. Kinderleben zwischen Straße und Schule. Weinheim/München: Juventa, 47-66.

Zinnecker, Jürgen (2001 [1990]): Vom Straßenkind zum verhäuslichten Kind. Kindheitsgeschichte im Prozeß der Zivilisation. In: Jürgen Zinnecker, Stadtkids. Kinderleben zwischen Straße und Schule. Weinheim/München: Juventa, 27-46.

Zinnecker, Jürgen (2012 [1978]): Recherchen zum Lebensraum des Großstadtkindes. Eine Reise in verschüttete Lebenswelten und Wissenschaftstraditionen. In: Imbke Behnken / Michael-Sebastian Honig (Hg.), Der Lebensraum des Großstadtkindes. Weinheim/Basel: Beltz Juventa, 19-74.

\section{Urban Childhoods between Utopia and Dystopia. Social and Educational Science Informed Rationalisations of the Topos "Children in the City"}

This article takes an analytical look at how urban childhoods are rationalised in social and educationally informed discourse about children in the city. This is done by elaborating the specific modes of rationalisation from the speech that discursively relate "children" and "city" to each other and ultimately position them topologically between utopia and dystopia via this. Guided by this discourse topological perspective, after a brief introduction the topos will first be considered as an explicit object of empirical, social and educational research, before essential stages of the speech about children in the city will be traced in the form of chronological highlights. Following this, street childhood and the pedagogical province are analysed as multilayered and central narratives of this speech. Concluding thoughts reintroduce the present analysis to the speech and reflect on the topos "children in the city" as a kind of "discursive heterotopia". 